\section{SAT0055 SIMULATING THE PATHOGENESIS OF ARTHRITIS IN VITRO BY DEVELOPING A HUMAN-BASED MULTICOMPONENT 3D JOINT MODEL}

A. Damerau ${ }^{1}$, Y. Chen ${ }^{1}$, A. Lang ${ }^{1}$, I. Ponomarev ${ }^{2}$, M. Pfeiffenberger ${ }^{1}$, F. Buttgereit ${ }^{1}, \mathrm{~T}$. Gaber ${ }^{1} .{ }^{1}$ Department of Rheumatology and Clinical Immunology, Charité-Universitätsmedizin Berlin, Berlin; ${ }^{2} f z m b \mathrm{GmbH}$, Bad Langensalza, Germany

Background: Our ultimate goal is to develop a valid human in vitro 3D joint model to simulate the pathogenesis of arthritis. The in vitro $3 \mathrm{D}$ joint model consists of different components including an ${ }^{1}$ osteogenic and ${ }^{2}$ chondrogenic part, ${ }^{3}$ the synovial fluid $a^{4} d^{4}$ the synovial membrane and contains all involved cell types and thus, to allow interactions between cells by cell contacts and signalling molecules. As an alternative experimental setup for animal models, our in vitro 3D joint model will enable us to study the influence and efficacy of drug treatment. Currently, there is no valid 3D model which is able to mimic an arthritic joint.

Objectives: Here, we aim to mimic the ${ }^{1}$ osteogenic and $d^{2}$ chondrogenic part, ${ }^{3}$ the joint space with synovial fluid and ${ }^{4}$ the synovial membrane.

Methods: For the osteogenic component of the 3D joint model, we populated $\beta$ tricalcium phosphate (TCP) - mimicking the mineral bony part - with osteogenic pre-differentiated human bone marrow-derived mesenchymal stromal cells (hMSC) and coated the particles with an hMSC monolayer cell-sheet to get a compact bony component. Survival, adhesion and structural integrity of the cells were evaluated by Scanning Electron Microscopy (SEM), LIVE/DEAD staining and cellular release of $\mathrm{LDH}$. Osteogenic differentiation was analysed by $\mu \mathrm{CT}$ for mineralization and on gene expression level using qRT-PCR. To mimic the chondrogenic part, a scaffold-free 3D cartilage construct was generated by chondrogenic differentiation of hMSC under hypoxia with intermittent mechanical stimulation. Constructs were analysed by histology and qRT-PCR. Simulating the synovial fluid, hyaluronic acid was applied to the osteochondral model. To model the synovial membrane, a confluent monolayer of hMSC was formed on a polycarbonate membrane and visualised by hemacolor staining.

Results: We developed an in vitro 3D bone model by successfully seeding predifferentiated hMSC on a $\beta$-TCP scaffold. Cells consistently adhere onto the scaffold for up to 3 weeks as observed by SEM. The analysis of cell viability via LDH detection and LIVE/DEAD staining showed no toxic effects on the cells even after 3 weeks of incubation as compared to the corresponding control. mRNA expression of bone-related genes such as RUNX2, SPP1 and COL1A1 as well as $\mu C T$ analysis confirmed the osteogenic phenotypic of hMSC grown in 3D. Mimicking the articular cartilage component, we verified its chondrogenic phenotype by $\mathrm{HE}$ and Alcian Blue staining as well as by the reduced mRNA expression of COL1A1 and an abundant expression of COL2A1. Interestingly, co-cultivation of the osteogenic and chondrogenic part for up to 3 weeks demonstrated successful colonisation, connectivity and initial calcification implying a functional transitional bridging area. Modelling the synovial membrane, we successfully and reproducibly created a confluent monolayer of hMSC, which is easily transferable to the model. Conclusions: First steps towards the in vitro simulation of an arthritic joint based on a multi-component model confirm good cell vitality and phenotypic stability which indicates successful progression. To finalise the development of healthy joint model, we will combine the established parts to provide a suitable 3D multicomponent joint model which enables us to study the efficacy of drug treatment in vitro.

Disclosure of Interest: None declared

DOI: 10.1136/annrheumdis-2018-eular.3248

\section{SAT0056 OSTEOARTHRITIS SEVERITY IS REDUCED BY INTRA- ARTICULAR ADMINISTRATION OF HYDROGEN SULFIDE}

E.F. Burguera $^{1,2}$, Á. Vela-Anero ${ }^{3}$, T. Hermida-Gómez ${ }^{1}$, P. Filgueira-Fernández ${ }^{1,2}$, L. Gato-Calvo ${ }^{1}$, R. Meijide-Failde ${ }^{3}$, F.J. Blanco ${ }^{1} .{ }^{1}$ Rheumatology Research Group, Instituto de Investigación Biomédica de A Coruña, A Coruña; ${ }^{2}$ CIBER-BBN, Madrid; ${ }^{3}$ Cell Therapy and Regenerative Medicine Group, Dep. of Medicine, University of A Coruña, A Coruña, Spain

Background: Osteoarthritis $(\mathrm{OA})$ is a chronic inflammatory disease leading to cartilage loss and eventual joint destruction. Exogenous supplementation of hydrogen sulphide $\left(\mathrm{H}_{2} \mathrm{H}\right)$ with synthetic salts in in vitro models of $\mathrm{OA}$ has been shown to exert anti-inflammatory effects and to result in reduced cartilage degradation ${ }^{1}$

Objectives: To evaluate the effects of administering an $\mathrm{H}_{2} \mathrm{~S}$-producing compound intra-articularly in an experimental model of $O A$.

Methods: Experimental OA was induced in Wistar rats by transecting the medial collateral ligament and removing the medial meniscus of the left knee. Right knees were used as control. Animals were randomised into 3 groups ( 3 rats per group). Group 1 (intra-articular sulphide, IS): A single intra-articular injection of GYY4137
(200 $\mathrm{mM}$ in saline, $50 \mathrm{ml}$ ) at day 7. Group 2 (intra-articular control, IC): A single intra-articularly injection of vehicle (saline, $50 \mathrm{ml}$ ) at day 7. Group 3 (Surgical control, C): No treatment.

Gross evaluation of the animals at days 0 (before surgery), 7, 15 and 40 (euthanasia) included indirect evaluation of pain in a Rotarod performance test. Histopathological changes in articular cartilage and synovium were evaluated with the Mankin Score (MS) and the Krenn Score (KS), respectively.

Results: All 3 groups showed worse performance in the Rotarod test at day 7 after surgery. Number of falls was significantly increased (except in IC) and time to $1 \mathrm{st}$ fall was reduced (table 1). At day 40 , there was no significant improvement in either of these parameters in group C, while in IC the $\mathrm{n \#}$ of falls had returned to pre-surgical levels. In IS there were significant improvements with respect to day 0 and both $C$ and IC groups (table 1). Times to 1 st fall were also significantly better in the IS group vs. C and IC both at days 15 and 40 .

Histology showed no significant differences among groups in the lateral tibial plateau (TP) or femoral condyle (FC) separately or in the compartment as a whole. Conversely, MS in the medial compartment were significantly better in the IS group vs the $\mathrm{C}$ group, both when considering TP or FC separately, and for the whole compartment (figure 1). No significant differences were found among groups on the Krenn Scores.

Abstract SAT0056 - Table 1. Number of falls ( $\mathrm{n} \pm \mathrm{sd}$ ) and time to first fall (s) in a Rotarod. C: control group; IC: intra-articular control; IS: intra-articular sulphide ( $n=3 /$ group). $\# p<0.05$ vs. T0; ${ }^{*} p<0.05$ vs. C. ${ }^{\$} p<0.05$ vs. IC.

\begin{tabular}{ccccccccc}
\hline & \multicolumn{4}{c}{ Falls $(\mathrm{n} \pm \mathrm{sd})$} & \multicolumn{5}{c}{ Time to 1 st fall $(\mathrm{s})$} \\
\hline & T0 & T7 & T15 & T40 & T0 & T7 & T15 & T40 \\
\hline C & 0.8 & 4.0 & 4.0 & $3.7 \pm 1.7 \#$ & 233.7 & 57.2 & $90.3 \pm 67.5 \#$ & 77.8 \\
& \pm 0.7 & $\pm 0.9 \#$ & $\pm 0.9 \#$ & & \pm 77.8 & $\pm 35.2 \#$ & & $\pm 40.2 \#$ \\
IC & 1.7 & 3.3 & $2.2 \pm 1$. & $2.0 \pm 0.9$ & 209.2 & 31.7 & $90.8 \pm 20.9 \#$ & 143.3 \\
& \pm 1.0 & \pm 1.7 & 0 & & \pm 78.2 & $\pm 11.7 \#$ & & \pm 50.3 \\
IS & 1.3 & 4.0 & 2.5 & 0.0 & 265.3 & 78.5 & 196.3 & 300.0 \\
& \pm 0.8 & $\pm 1.5 \#$ & \pm 1.1 & $\pm 0.0 \#^{\star \$}$ & \pm 58.8 & $\pm 65.9 \#$ & $\pm 81.3^{* \$}$ & $\pm 0.0^{* \$}$ \\
\hline
\end{tabular}
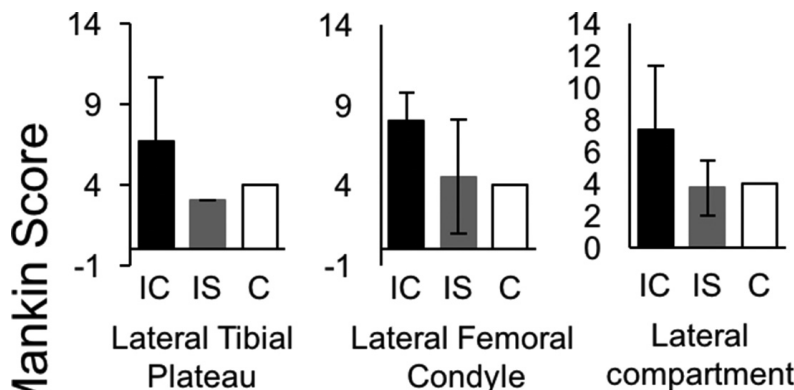

\section{Lateral Femoral}

Lateral

$\sum$
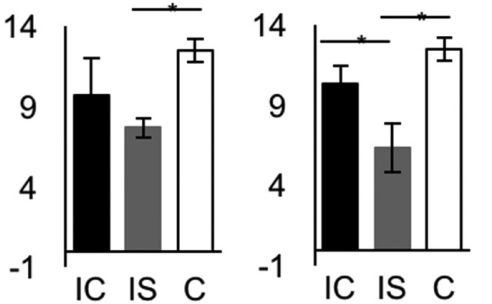

compartment

\section{Medial Tibial}
Medial Femoral Condyle

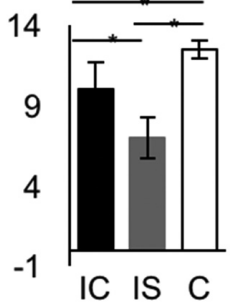

Disclosure of Interest: None declared

DOI: 10.1136/annrheumdis-2018-eular.3635

Conclusions: Exogenous $\mathrm{H}_{2} \mathrm{~S}$ administered intra-articularly (200 mM GYY4137 in $50 \mathrm{ml}$ saline) can reduce the severity of cartilage destruction in an in vivo model of $\mathrm{OA}$ as compared to no treatment or a vehicle control. $\mathrm{H}_{2} \mathrm{~S}$ also led to a reduction in pain levels as demonstrated by a performance test. Therefore, hydrogen sulphide is a viable pharmacological candidate for OA treatment and should be further tested, including human clinical trials.

\title{
REFERENCE:
}

[1] Vela-Anero A, et al. Long-term effects of hydrogen sulfide on the anaboliccatabolic balance of articular cartilage in vitro. Nitric Oxide 2017;70:42-50. 